\title{
Preface: Special Issue on Combinatorial Optimization
}

\author{
Xiao-Dong Hu ${ }^{1}$. Guo-Hui Lin ${ }^{2}$. Li-Ying Kang ${ }^{3}$. Guo-Chuan Zhang ${ }^{4}$ \\ Published online: 26 August 2019 \\ () Operations Research Society of China, Periodicals Agency of Shanghai University, Science Press, and \\ Springer-Verlag GmbH Germany, part of Springer Nature 2019
}

Combinatorics is, as defined by MathWorld, the branch of mathematics that studies the enumeration, combination and permutation of sets of elements and the mathematical relations that characterize their properties. Combinatorial optimization not only enumerates the sets studied, but also has the goal of finding the member of the set in an efficient manner that optimizes an objective function. Combinatorial optimization is an emerging field at the forefront of combinatorics, theoretical computer science and operations research that has various applications in many disciplines of science, engineering, industry, economy, military and so on.

Combinatorial optimization has experienced an explosive rate of growth since computers are widely used, particularly in the past two decades. This growth rate is partly due to the development of related research areas, such as data mining, communication networks, bioinformatics and computational game theory. These newly established research areas generate a large number of new, hard combinatorial optimization problems, most of which have direct applications to real-world problems, and so efficient solutions to them are actively sought after.

Some of the important results can be found in the Journal of Combinatorial Optimization published by Springer. Ding-Zhu Du has been the editor-in-chief of the journal since it was launched in 1997. He has made many important contributions in

\footnotetext{
$\bowtie$ Xiao-Dong Hu

xdhu@amss.ac.cn

Guo-Hui Lin

ghlin@cs.ualberta.ca

Li-Ying Kang

lykang@shu.edu.cn

Guo-Chuan Zhang

zgc@zju.edu.cn

1 Academy of Mathematics and Systems Science, Chinese Academy of Sciences, Beijing 100190, China

2 Department of Computer Science, University of Alberta, Edmonton, AB T6G 2R3, Canada

3 Department of Mathematics, Shanghai University, Shanghai 200444, China

4 College of Computer Science and Technology, Zhengjiang University, Houzhou 310027, China
} 
the past four decades to computational complexity, approximation design and analysis, graph theory, combinatorics and so on. This special issue is an exhibition of Du's great influence and inspiration to many young Chinese researchers. The topics in this collection encompass a broad area of combinatorial optimization algorithms and applications, including the design of approximation and online algorithms, matroid theory, algorithmic game theory, graph theory, optimization of (non-)submodular functions, scheduling problems, etc. These papers are summarized as follows.

Min Li, Dong-Lei Du, Da-Chuan Xu and Zhen-Ning Zhang studied the problem of maximizing a concave function over an intersection of two partition matroids in the paper entitled "A Note on Submodularity Preserved Involving the Rank Functions". They showed that submodularity may not be preserved, but it involves one maximal submodular problem (or minimal supermodular problem) with some conditions.

Yu-Kun Cheng and Zi-Xin Zhou studied the market equilibrium mechanism in resource sharing system in the paper entitled "An Improved Incentive Ratio of the Resource Sharing on Cycles". They proved that the incentive ratio on cycle networks is upper bounded by 3; the ratio measures how much a participant could gain from the Sybil attack by splitting its identity and reconstructing its communication connections with others.

Yao Xu, Yong Chen, Peng Zhang and Randy Goebel investigated the Maximum Happy Vertices (MHV) problem and its complement, the Minimum Unhappy Vertices (MUHV) problem in the paper entitled "Approximation Algorithms for Vertex Happiness". They showed MUHV problem is Unique Games-hard to achieve a (2$2 / k-\varepsilon$ )-approximation, for any $\varepsilon>0$. For the MHV problem, the $2 / k$-approximation improves the previous best approximation ratio $\max \{1 / k ; 1 /(\Delta+1 / \operatorname{g}(\Delta))\}$, where $\Delta$ is the maximum vertex degree of the input graph and $g(\Delta)>4 \Delta^{2}$.

Yi-Jing Wang, Da-Chuan Xu, Yan-Jun Jiang and Dong-Mei Zhang investigated the problem of minimizing the ratio of normalized non-negative monotone nonsubmodular set function $f$ and normalized non-negative monotone set function $g$ in the paper entitled "Minimizing Ratio of Monotone Non-Submodular Functions". They obtained a performance guarantee depending on the generalized curvature and inverse generalized curvature of $f$, as well as the submodularity ratio of $g$.

Daniel Li Li and Er-Fang Shan studied the cooperative games under the partial cooperation structures described by graphs in the paper entitled "The Myerson Value on Local Structures of Coalitions". They proposed an axiomatic characterization of the Myerson value associated with local cooperative structures.

Yong Chen, Guang-Ting Chen, Long-Cheng Liu, Yi-Wei Jiang, Zhi-Yi Tan and An Zhang investigated online scheduling problems with processing set restrictions in the paper entitled "Online Scheduling with Unit Processing Times and Processing Set Restrictions". They proposed an optimal algorithm with a competitive ratio of $3 / 2$ for $m$ machines with two different processing sets, and an optimal algorithm with a competitive ratio $5 / 3$ for three machines with inclusive processing sets.

Long Wan and Jin-Jiang Yuan studied the approximation of all feasible schedules in a given scheduling environment by one schedule in the paper entitled "Simultaneous Approximation Ratios for Parallel-machine Scheduling Problems". They introduced the concepts of strong simultaneous approximation ratio and weak simultaneous 
approximation ratio and studied the two variants under various scheduling environments.

Hui Shang, Eminjan Sabir and Ji-Xiang Meng studied the $k$-component edge connectivity in the paper entitled "Conditional Connectivity of the Locally Twisted Cubes". They determined the component edge connectivity of the locally twisted cubes and characterized the corresponding optimal solutions.

All the papers in this special issue have been peer reviewed with the standard of the journal. Our deep and sincere gratitude goes to all the authors for their timely response and careful revisions. They have made tremendous contributions and offered generous support to this issue. We also thank the anonymous referees for their voluntary work and expert reviews.

July 2019 